1 Universidade de São Paulo (USP), Faculdade de Medicina, Departamento de Medicina Preventiva São Paulo (SP), Brasil. paulohsmota@gmail.com

2 Universidade de São Paulo (USP), Faculdade de Medicina, Departamento de Medicina Preventiva - São

Paulo (SP), Brasil.

analuizaviana@usp.br

3 Universidade de São Paulo (USP), Faculdade de Saúde Pública, Departamento de Prática de Saúde Pública São Paulo (SP), Brasil. aylenebousquat@usp.br

\section{Relações federativas no Programa Academia da Saúde: estudo de dois municípios paulistas}

\author{
Federative relations at the Programa Academia da Saúde (Health \\ Academy Program): study of two cities of the state of São Paulo
}

Paulo Henrique dos Santos Mota'1, Ana Luiza d'Ávila Viana², Aylene Bousquat ${ }^{\mathbf{3}}$
RESUMO O objetivo é a análise das relações federativas na implementação do Programa Academia da Saúde em municípios do estado de São Paulo. Realizado estudo de caso com análise documental e entrevistas semiestruturadas; utilizou-se o referencial teórico do ciclo da política. Observou-se indução direta do Ministério da Saúde no município por meio da normalização, dos interesses políticos e do incentivo financeiro. A participação dos estados é burocrática e restrita à Comissão Intergestora Bipartite. Municípios são financeiramente dependentes para implementação. Não se observa possibilidade concreta de adaptação às realidades locais.

PALAVRAS-CHAVE Política de saúde; Federalismo; Promoção da Saúde.

ABSTRACT The aim is the analysis of federal relations in the implementation of the Programa Academia da Saúde (Health Academy Program) in municipalities of the state of São Paulo. We conducted a case study with documental analysis and semi-structured interviews; we used the theoretical framework of the Policy Cycle. There was direct induction of the Ministry of Health in the municipality through standardization, political interests and financial incentive. The participation of the states is bureaucratic and restricted to the Comissão Intergestora Bipartite. Municipalities are financially dependent for implementation. It has not been seen a concrete possibility of adjustments to the program to local and regional realities.

KEYWORDS Health policy; Federalism; Health Promotion. 


\section{Introdução}

A Constituição Federal de 1988 tornou a saúde responsabilidade dos três níveis governamentais. O Sistema Único de Saúde (SUS) foi formado a partir dos princípios da universalidade, equidade e integralidade (NORONHA; LIMA; MACHADO, 2008). Cabem aos três níveis de governo (União, estados e municípios) a gestão e o financiamento do SUS, de modo a garantir a atenção à saúde segundo esses princípios (ELIAS; DOURADO, 2011).

O modelo assistencial prévio voltado preponderantemente para processos curativos foi modificado pelo SUS. Ganha destaque a atenção ao indivíduo, e não à doença, bem como a coordenação e integração dos serviços prestados. A estratégia utilizada para a realização e implantação das ações em saúde ocorreu por meio da descentralização, iniciando-se pela estadualização da gestão, avançando nos anos de 1990 para a municipalização da gestão de ações e serviços em saúde (VASCONCELOS ET AL., 2009).

O município ganhou destaque, com papel central da gestão na saúde, principalmente no que se refere à Atenção Básica $(\mathrm{AB})$. Apesar de trazer resultados positivos, persistiram problemas decorrentes da intensa fragmentação do sistema, resultando em pequenos sistemas locais que expandiram o acesso à população, mas não conseguiram orientar e ordenar os serviços de saúde de forma a alterar as desigualdades regionais, $o$ acesso e a utilização dos serviços de saúde, bem como o insuficiente gasto público em saúde. Além disso, não houve integração de serviços, instituições e práticas nos territórios nem a formação de arranjos mais cooperativos no setor (VIANA; MACHADO, 2009).

A implantação dessas políticas, em cenários de flexibilidade institucional, foi acompanhada da criação de centros decisórios autônomos e interdependentes, alcançada pelo esforço de profissionais nos diferentes níveis de governo, por meio da criação de novos critérios para alocação e transferência de recursos, novas instâncias de negociação, envolvendo a participação dos gestores, prestadores de serviços, profissionais de saúde e usuários, por intermédio dos Conselhos de Saúde e das Comissões Intergestoras Tripartite (CIT) e Comissões Intergestoras Bipartite (CIB) (VASCONCELOS ET AL., 2009).

Com a publicação da Portaria ${ }^{\circ}$ 95, de 26 de janeiro de 2001, que instituiu a Norma Operacional da Assistência à Saúde (Noas), a esfera estadual ganha uma nova importância. São criados instrumentos de planejamento regional envolvendo atividades de assistência à saúde, organizando recursos e responsabilidades pelos três entes federados. Estabeleceram-se mecanismos de referência e contrarreferência de forma a garantir a integração de serviços e ações e a integralidade assistencial. Priorizaram-se estratégias voltadas para medidas preventivas, realizadas por meio de ações educativas para o controle dos condicionantes de risco (ALBUQUERQUE, 2014).

O Pacto pela Saúde, lançado no ano de 2006, nasceu acompanhado de um compromisso público dos gestores do SUS para sua sustentação, sendo reestabelecido ano a ano. A questão essencial abordada pelo Pacto trata de uma rede de coordenação entre os entes federativos, apontando para a necessidade de melhorar e estruturar uma maior coordenação e cooperação entre os três entes federados. Deu-se ênfase aos desafios prioritários para a saúde da população, sendo articulados em três componentes que iriam guiar o compromisso: Pacto pela Vida, Pacto em Defesa do SUS e o Pacto de Gestão do SUS (AlbUQUeRQue, 2014).

Com o lançamento do Decreto Presidencial n 7.508 em 2011, que regulamenta a Lei $\mathrm{n}^{\circ} 8.080$, de 1990 , o processo de regionalização do SUS ganha centralidade na política de saúde. Reafirma-se a AB como ordenadora do cuidado. Define-se que o acesso universal e igualitário deve ser orientado dentro de uma rede regionalizada 
e hierarquizada. O planejamento das ações passa a ser ascendente e realizado de forma integrada entre União, estado e município, baseado nas diretrizes formuladas pelos Conselhos de Saúde (municipais, estaduais e nacional), levando-se em conta as necessidades de saúde e a disponibilidade dos recursos financeiros e pactuado das CIB e CIT.

Os atores provenientes de tais comissões e conselhos passam a tomar parte nas definições regionais, tendo em vista o caráter federativo do processo de descentralização da área, e se associam a esses representantes da sociedade, gerando assim uma maior participação popular no processo de tomada de decisão (VIANA; LEVCOCITZ, 2005).

De forma concomitante às questões relacionadas com a gestão da saúde, o Ministério as Saúde trabalha em ações voltadas para a prevenção das Doenças Crônicas Não Transmissíveis (DCNT) (BRASIL, 2010, 2011) dentro de um contexto do aumento dessas e da diminuição de incidência de doenças infectocontagiosas (SCHMIDT ET AL., 2011).

Com essas preocupações e inspirado em experiências exitosas levadas a cabo em Minas Gerais e Pernambuco, o Ministério da Saúde lança, em abril de 2011, a Portaria $n^{0}$ 719, que cria o Programa Academia da Saúde (PAS) (COSTA ET AL., 2013; HALLAL ET AL., 2010). Este tem como objetivo principal contribuir para a promoção da saúde da população assistida por meio da implementação de um polo com infraestrutura adequada e recursos humanos qualificados. Prevê a prática intersetorial em suas ações, participação popular, além de ser parte do cuidado integral ao usuário.

Deverão ser estruturados 'espaços saudáveis’ que devem promover ações de promoção da saúde de forma intersetorial, estimulando a atividade física/práticas corporais, o lazer, modos de vida e práticas alimentares saudáveis em determinado território, em articulação com a rede de Atenção Básica à Saúde.
O presente trabalho tem como objetivo analisar as relações federativas na implementação do PAS em municípios selecionados do estado de São Paulo.

\section{Método}

O referencial teórico utilizado foi o ciclo da política, a partir da perspectiva de um estudo de caso, sendo utilizada a abordagem qualitativa. Tal ciclo subdivide o processo político em cinco fases: (1) montagem da agenda; (2) formulação da política; (3) tomada de decisão; (4) implementação da política e (5) avaliação da política. Esse visa a representação de um complexo processo envolvendo diferentes etapas e tem como objetivos centrais questionar a ação pública, seus determinantes, finalidades, processos e consequências (HOWLETT; RAMESH; PERL, 2013).

A principal contribuição do ciclo da política é a percepção da existência de diferentes momentos no processo de construção de dada política. Leva-se em conta a necessidade de reconhecer as especificidades de cada um desses momentos (BAPTISTA; REZENDE, 2011).

O estudo de caso trata de investigar um fenômeno contemporâneo em um contexto de vida real, estando os limites entre o fenômeno e o contexto não claramente evidentes. A escolha dessa modalidade de estudo ocorreu pela possibilidade de investigar questões empíricas no contexto atual; explorar situações da vida real e explicar as variáveis causais do fenômeno estudado diante das situações complexas referentes à tomada de decisões e seus possíveis resultados. Dessa forma, o estudo de caso fornece conhecimento aprofundado de uma realidade delimitada, sem existir, por parte do pesquisador, controle dos eventos comportamentais (GIL, 2002). Para tal, foram definidas duas formas de extração de dados: análise documental e entrevistas semiestruturadas. 
A análise documental buscou compreender estudos de base normativa, tais como: Decretos e Portarias para a política de inserção e construção do PAS, assim como atas das reuniões das CIB e notas técnicas.

As entrevistas foram realizadas com atores-chave municipais, que participaram dos processos de montagem da agenda, formulação da política, tomada de decisão e implementação da política. Foram realizadas com secretários municipais de saúde, coordenadores de Atenção Básica (AB), coordenador de práticas integrativas e complementares e gestor do polo PAS. Como critério de inclusão para entrevista, foi utilizado o período de ao menos seis meses na função.

Foram selecionados dois municípios, utilizando-se os seguintes critérios: 1) municípios que aderiram ao programa por intermédio de critérios de similaridade como previsto na Portaria $n^{\circ} 1.402$, de 15 de junho de 2011 - entende-se dessa forma que, por estarem atuando há mais tempo, a aprendizagem dos gestores e profissionais em lidarem com as problemáticas envolvidas na estruturação e implementação do programa poderiam suprir as perguntas da pesquisa; 2) pertencerem a diferentes regiões de saúde estabelecidas a partir do Decreto Federal no 7.508 de junho de 2011, que regulamenta o processo de regionalização da saúde no Brasil.

As entrevistas semiestruturadas foram gravadas e transcritas para meio eletrônico, procedendo-se análise a partir de leitura geral dos textos e subsequentes aproximações sucessivas. Dessa forma foi possível identificar elementos de maior recorrência e relevância a partir da visão dos atores participantes. Organizou-se os temas em duas dimensões e duas subdimensões: 1) contexto político e histórico da entrada na agenda - configuração histórica de promoção da saúde, problematização da implementação; quando e como se configurou a proposta das Academias da Saúde - e 2) dinâmica de ação dos entes federativos - participação entes federativos na implementação, mudanças e inflexões relativas às iniciativas federais e dinâmica entre os entes na coordenação de prevenção e promoção. Por fim, foi realizada verificação de existência ou não de convergência entre as respostas dos atores entrevistados.

O estudo foi aprovado pelo Comitê de Ética da Faculdade de Medicina da Universidade de São Paulo com protocolo de número 21553114.1.0000.0065. Os municípios não serão identificados devido à solicitação de um dos gestores entrevistados.

\section{Resultados e discussão}

O presente estudo teve como ponto de partida o caso de dois municípios do estado de São Paulo. O município A, de grande porte, localiza-se na região de saúde do Alto do Tietê, e o município $\mathrm{B}$ pertence à região do Vale do Paraíba/Região Serrana sendo de pequeno porte.

$O$ relativo curto período entre o lançamento da Portaria $n^{0} 719$, de abril de 2011, dando início ao PAS, e o desenvolvimento da presente pesquisa levou à restrição dos casos estudados. Esses primeiros municípios podem traduzir uma expressão de como o programa a curto prazo aponta problemas e respostas, possibilitando, assim, ajustes para uma possível reentrada no processo do ciclo da política e a maturação do programa.

Por outro lado, um estudo realizado com maior tempo de implementação do programa possibilitaria uma maior diversidade de municípios, gerando um maior entendimento da realidade do processo de implementação. Ainda assim, a inclusão de dois municípios com diferentes características socioeconômicas, geográficas e populacionais permitiu verificar a interação do ciclo da política pública nos contextos locais.

Foram entrevistados os seguintes 
gestores dos dois municípios: secretário municipal de saúde, secretário adjunto de saúde, coordenador de $\mathrm{AB}$, coordenador de práticas integrativas e complementares e gestor do polo.

No município A, tanto o secretário adjunto de saúde como o coordenador de $\mathrm{AB}$ se encontram em seus cargos há mais de dois anos. O coordenador de práticas integrativas e complementares exerce a função em um período entre 13 e 24 meses, e o gestor do polo PAS encontra-se no cargo há oito meses, sendo anteriormente coordenador de Unidade Básica de Saúde (UBS).

No município B, o gestor se encontra no cargo há mais de quatro anos, acumulando a função de gestor do polo PAS desde o momento de sua instituição, no ano de 2011. Segundo este, não existe necessidade de contratação de um profissional específico para a função, uma vez que se trata de um município de pequeno porte, onde são escassos os recursos financeiros, e as demandas como secretário de saúde permitem que acumule tal função.

\section{Entrada na agenda e formulação da proposta}

Políticas de promoção da saúde começam a surgir de forma mais incipiente na agenda do governo federal a partir dos anos 2000 . Concomitantemente, gestores locais criam ações voltadas para promoção de atividade física, prevenção de violência, redução da mortalidade no trânsito, entre outras (MALTA ET AL., 2014).

Com a inclusão na agenda federal entre os anos de 2008-2011, por meio de agregação no Pacto pela Vida, a Política Nacional de Promoção à Saúde e a publicação do Plano Nacional de Enfrentamento das DCNT, o tema ganha maior destaque, com foco no combate ao tabagismo, alimentação inadequada, inatividade física e consumo excessivo de bebidas alcoólicas (BRASIL, 2006, 2010, 2011;
MALTA ET AL., 2014).

O lançamento do PAS eleva a preocupação com tais fatos a um programa em desenvolvimento e implementação. Fato esse realizado, segundo nota técnica 16/2011 do Conselho Nacional de Secretários de Saúde (Conass), sem pactuação pelos gestores do SUS. O tema somente foi abordado nos grupos de trabalho de Atenção à Saúde e de Vigilância à Saúde da CIT ao se propor os fatos para a operacionalização do programa, não havendo, portanto, qualquer discussão nessa instância a respeito de sua formulação.

Segundo secretário adjunto do município A, o PAS entra na agenda e é formulado no seio das políticas nacionais voltadas à promoção da saúde/intervenções relacionadas com atividades físicas e mudanças de hábitos alimentares. São implementadas não somente visando ao alcance de parcela populacional sujeita às interferências dos fatores de risco, mas também visando a formas de promoção da saúde conduzidas em diferentes níveis (individual, grupos específicos e populações), contextos (clínicas, escolas, local de trabalho e mídia) e abordagens (aconselhamento individual, educação, regulamentos, modificações ambientais).

\section{Tomada de decisão e a implementação do PAS}

A Portaria $\mathrm{n}^{\circ} 719$, de abril de 2011, trata que, nos processos de formulação, tomada de decisão e implantação do programa, cada um dos entes federativos (União, estados e municípios) tem compromissos e características próprias de atuação.

O planejamento ocorre prioritariamente por ações do Ministério da Saúde envolvendo aspectos, como a entrada na agenda de discussão política, tomada de decisão, proposição de intervenções, identificação de necessidades, elaboração de estratégias e articulação com atores, para operacionalizar determinada política ou programa. 
Sua influência é acompanhada por meio de métodos de indução utilizados pelo Ministério da Saúde sobre os municípios que irão aderir ao programa.

Tais métodos indutivos ocorrem por meio da divulgação de Portarias relativas às diferentes políticas e programas estabelecidos e, sobretudo, na forma de incentivos financeiros para a implantação e para o custeio do programa em cada município.

Foram apontadas as duas formas de indução federal. Em ambos os casos, houve o processo indutivo por meio de portarias, contemplando critérios de similaridade com programas previamente existentes, por portaria e por intermédio de custeio. Os casos estudados ainda apresentam polos do programa construídos ou em construção por incentivo federal.

O município A recebe incentivo para a construção de 11 polos, e o município B, para a construção de um polo. Em ambos os casos, um polo foi instituído por critérios de similaridade com programas previamente estabelecidos que apresentavam objetivos comuns ao PAS.

Ao avaliar nota técnica de número 22/2012 do Conass, nota-se que os critérios de seleção das cidades contempladas como prioridades foram estabelecidos pelo Ministério da Saúde sem qualquer processo de pactuação ou discussão tripartite. Tais critérios foram: porte populacional, condição de vulnerabilidade social (população em situação de extrema pobreza) e proporção de propostas cadastradas por unidade federativa.

Segundo informações apontadas pelos gestores municipais dos casos analisados, o processo de indução federal-municipal se acentua pela pouca presença da figura do governo do estado na tomada de decisão e implementação de programas federais. A figura do ente estadual não é presente no processo de articulação do programa.

O envolvimento estadual com o PAS ocorre de forma estritamente burocrática, ou seja, é um mecanismo de passagem de informações e autorizações, uma vez que essas, advindas do governo federal para a implementação, ou qualquer outra alteração dentro do plano de trabalho do programa, deve ter o consentimento da CIB, assim como da Divisão Regional de Saúde (DRS) referente ao município, no caso do estado de São Paulo.

Esse fato também foi observado quando da análise das atas das reuniões da CIB do estado de São Paulo, no período de abril de 2011 (mês em que foi promulgado o primeiro decreto referente ao Programa) a outubro de 2014. Nessas atas, não há relato de discussão sobre quaisquer questões relativas ao Programa. Desse modo, das 42 atas analisadas no período acima citado, 27 (64,3\%) apresentam alguma informação referente ao PAS. Em todas essas atas, os temas abordados são aprovações para solicitação de verbas para construção e/ou custeio (43\%), mudanças de endereços (24\%), solicitação de emenda parlamentar para a construção do polo (29\%), informes (2\%) e mudanças de convênio com o Ministério da Saúde (2\%); confirmando que o envolvimento para estabelecimento do programa é realizado de forma ativa somente nas esferas federal e municipal.

$\mathrm{Na}$ implementação do PAS, os gestores locais afirmam que o processo de pactuação e articulação entre União e municípios é frágil, uma vez que ocorre de forma normativa e financeira com pouco ou nenhum apoio para a formação técnica do profissional e integração à rede de atenção à saúde.

Ao estudar os contextos federativos para a implantação da Rede Amamenta Brasil em Porto Alegre (RS), Corumbá (MS) e no Distrito Federal (DF), Venâncio et al. (2013) apresentam no contexto federal um caráter homogêneo de influência para a implantação de programas por meio de portarias federais e aporte de recursos financeiros. No nível estadual, relatam uma dificuldade de diálogo entre os diferentes municípios, 
assim como a priorização de programas próprios. Finalmente, no contexto municipal, apontam o apoio com recursos financeiros em um dos municípios como diferencial positivo para a implantação da política, e como um problema que dificulta seu estabelecimento a concorrência com outros programas similares e a distância entre o implantador da política de sua execução e coordenação.

A implantação do PAS em um município de grande porte aumenta a burocracia e pode vir a impedir a autonomia do gestor responsável pelo serviço. Tal problema não ocorre em município de pequeno porte, uma vez que a proximidade física leva a um maior entendimento das necessidades para a execução de uma determinada ação.

O financiamento é apontado como o principal fator de dificuldade para implementação, execução e expansão do programa. São repassados, pelo Ministério da Saúde, recursos na forma de custeio, voltados para a contratação e pagamento dos profissionais que atuam no programa.

São duas as formas de transferência de recursos, ambas na modalidade fundo a fundo. A primeira ocorre por meio de repasses via Piso da Atenção Básica Variável, e somente ocorre quando existe vínculo entre o polo do PAS e um Núcleo de Apoio a Saúde da Família (Nasf), sendo que cada Nasf pode ter somente três polos do programa vinculados. A segunda forma decorre da transferência fundo a fundo financiada pelo Piso Variável da Vigilância e Promoção da Saúde, mediante vínculo do polo com uma UBS. O quadro 1 apresenta maiores detalhes sobre as transferências de recursos.

Quadro 1. Tipos de incentivo recebidos para implementação do PAS. 2014

\begin{tabular}{l|l|l}
\hline \multicolumn{1}{c|}{$\begin{array}{c}\text { Incentivos PAB } \\
\text { Variável }\end{array}$} & \multicolumn{1}{c}{ Valor } & Condições para recebimento do recurso \\
\hline Atenção Básica & $\begin{array}{l}\text { R\$3.000 mensais } \\
\text { por polo }\end{array}$ & $\begin{array}{l}\text { 1. Possuir Nasf } \\
\text { 2. Possuir polo do PAS construído com o incentivo de investi- } \\
\text { mento do Ministério da Saúde, devendo o polo estar vinculado } \\
\text { a esse Nasf; } \\
\text { 3. Possuir programa habilitado como iniciativa similar ao PAS, } \\
\text { habilitado em portaria do MS. } \\
\text { 4. Serão custeados somente três polos por Nasf. }\end{array}$ \\
\hline $\begin{array}{l}\text { Vigilância e Promoção em } \\
\text { Saúde } \$ 36.000 \text { anual por } \\
\text { município }\end{array}$ & $\begin{array}{l}\text { 1. Não ter Nasf no município. } \\
\text { 2. Possuir polo do PAS construído com o incentivo de investi- } \\
\text { mento do Ministério da Saúde. } \\
\text { 3. Possuir programa habilitado como iniciativa similar ao PAS, } \\
\text { habilitado em portaria do MS. }\end{array}$ \\
\hline
\end{tabular}

Todos os gestores municipais enxergam limites financeiros e ausência de autonomia para a implantação e manutenção do programa. As principais dificuldades residem no pagamento de profissionais capacitados e em melhorias e reformas de infraestrutura. Esse cenário gera dependência do nível federal para continuidade do programa. Não há menção ao ente estadual, pois, como afirmado acima, sua ação ocorre somente no nível formal.
Soares e Paim (2011), ao estudarem aspectos relacionados com a implantação da Política de Saúde Bucal em Salvador (BA), apresentaram informações que vão ao encontro dos achados do presente estudo, relacionando a implantação dessa política à ausência de autonomia financeira por parte do município, tornando-o dependente de recursos dos outros entes federativos para sua resolução.

Tal dependência financeira é abordada por Machado (2012) como um dos modelos 
adotados nos primórdios do SUS e intensificados a partir dos anos 2000, para indução de programas e políticas, juntamente ao planejamento e regulação de ações estratégicas. Esse modelo começa a partir de 2003 a sofrer grande número de críticas devido à normalização e burocratização das relações intergovernamentais. A forma de alteração dessa prática, em que existe a ênfase na indução, é apresentada no Pacto pela Saúde com a proposição de definir responsabilidades coletivas entre os entes federados, ou seja, em uma estrutura lógica em que existe apoio intergovernamental (LIMA; QUEIROZ, 2012).

Ao acentuar as proposições da Noas, o Decreto $\mathrm{n}^{0} 7.508$ concede maiores poderes e responsabilidades ao ente estadual por meio de operacionalização, financiamento e administração compartilhada dos programas e ações. Estabelece responsabilidades individuais e solidárias para a construção da rede de atenção à saúde. Em contrapartida, o Ministério da Saúde mantém propostas de articulação fundamentada na relação União-município, sendo induzidas de forma prévia ao Decreto (MACHADO, 2012).

Ao estudar o PAS, apresentado em 2011, poucos meses antes do lançamento do Decreto 7.508, a relação e o apoio intergovernamental aparecem fragilizados, sobretudo na figura dos estados da federação. A relação União-município se mantém, como relatado anteriormente, ao Decreto $\mathrm{n}^{\mathrm{o}} \mathbf{7 . 5 0 8}$, em que portarias e incentivos financeiros apresentam o tom para o processo de implementação com foco na descentralização para as esferas municipais (ALBUQUERQUE, 2014).

Constata-se a manutenção de uma estratégia de financiamentos por parte do Ministério da Saúde, em que a regularidade das transferências decorre da fidelização das esferas subnacionais à política induzida. A União mantém o poder de decisão na forma pela qual os recursos podem ou não ser aplicados, expressando suas prioridades (MACHADO, 2012).

No contexto de indução federal e de baixo apoio intergovernamental, a necessidade de articulação e de pactuação entre diferentes esferas de gestão governamental do SUS é uma questão central para a consolidação do sistema (VIANA; MACHADO, 2009).

Baptista (2007), em artigo de análise das portarias ministeriais, no período entre 1990 e 2002, aponta as ações do Ministério da Saúde como práticas centralizadoras e verticais, por meio da utilização de instrumentos indutivos que submetem os níveis subnacionais, principalmente os municípios. Segundo a autora, tal modalidade de relacionamento acaba acomodando as esferas municipais e, principalmente, as estaduais, uma vez que o Ministério sustenta suas políticas com garantias, recursos e benefícios.

Uma vez articuladas, as ações do PAS na rede de atenção municipal e no poder político poderiam minimizar as dificuldades de integração com outros serviços, reforçando o conceito de integralidade, estendendo a oferta organizada de serviços de assistência e prevendo garantias de referência e contrarreferência na rede de atenção. $\mathrm{O}$ desenvolvimento de tais articulações poderia levar a um estágio mais avançado de organização entre os diversos níveis e/ou equipamentos de atenção (GIOVANELLA ET AL., 2002).

\section{Conclusão}

O PAS entra na agenda federal a partir da mudança epidemiológica e demográfica da população brasileira. Esse ente acaba por induzir os municípios a implementar programas a partir da lógica federal, por meio de normalização e, principalmente, da disponibilidade de incentivos financeiros.

Sua implantação ocorre de forma indutiva pelo Ministério da Saúde diretamente aos municípios, havendo a participação dos estados de forma discreta, utilizando a CIB como instrumento meramente burocrático.

É, portanto, incongruente com as propostas de gestão propostas tanto pelo Decreto 
$\mathrm{n}^{\circ}$ 7.508, de 2011, quanto pelo Pacto pela Saúde em que se almeja construir uma responsabilização coletiva entre os três entes federativos sobre os programas e políticas implantados no âmbito do SUS.

O PAS, assim como outros programas recentes, é introduzido em um contexto de mudanças de relações entre os entes federativos, e por se basear em programas de

\section{Referências}

ALBUQUERQUE, M. V. The regional focus in Brazilian health policy (2001-2011): national guidelines and the regionalisation process in the states. São Paulo: USP, 2014.

BAPTISTA, T. W. F. Analysis of rulings by the Brazilian Ministry of Health and reflections on national health policy management. Cad. Saúde Pública, Rio de Janeiro, v. 23 , n. 3, p. 615-626, mar. 2007.

BAPTISTA, T. W. F.; REZENDE; M. A ideia de ciclo na análise de políticas públicas. In: MATTOS, R. A.; BAPTISTA, T. W. F. (Org.). Caminhos para análise das políticas de saúde. Rio de Janeiro: IMS, 2011.

BRASIL. Ministério da Saúde. Diretrizes operacionais dos pactos pela vida, em defesa do SUS e de gestão. Brasília, DF, 2006.

Ministério da Saúde. Plano de ações estratégicas para o enfrentamento das Doenças Crônicas Não Transmissíveis (DCNT) no Brasil 2011-2022. Brasília, DF, 2011.

Ministério da Saúde. Política nacional de promoção da saúde. Brasília, DF, 2010.

COSTA, B. V. L. et al. Academia da cidade: um serviço de promoção da saúde na rede assistencial do Sistema Único de Saúde. Ciênc. Saúde Coletiva, Rio de Janeiro, v. 18, n. 1, p. 95-102, jan. 2013.

ELIAS, P. E. M.; DOURADO, D. A. Sistema de saúde e SUS: saúde como política social e sua trajetória no iniciativas focalizadas, configura-se como uma ação que ainda é implantada como aqueles iniciados na década de 1990 no contexto da descentralização, por meio de forte indução federal ao município, por meio de incentivos e recursos financeiros para subsidiar uma agenda federal, não havendo possibilidade de reais adaptações do programa em relação às realidades locais e regionais.
Brasil. In: IBANEZ, N.; ELIAS, P. E. M.; SEIXAS, P. H. D. (Org.). Política e gestão pública em saúde. São Paulo: Hucitec, 2011.

GIL, A. C. Como elaborar projetos de pesquisa. São Paulo: Atlas, 2002.

GIOVANELLA, L. et al. Sistemas municipais de saúde e a diretriz da integralidade da atenção: critérios para avaliação. Saúde em Debate, Rio de Janeiro, v. 26, n. 60, p. 37-61, jan./abr. 2002.

HALLAL, P. C. et al. Avaliação do programa de promoção da atividade física Academia da Cidade de Recife, Pernambuco, Brasil: percepções de usuários e não-usuários. Cad. Saúde Pública, Rio de Janeiro, v. 26, n. 1, p. 70-78, jan. 2010.

HOWLETT, M.; RAMESH, M.; PERL, A. Política pública: seus ciclos e subsistemas. Rio de Janeiro: Elsevier, 2013.

LIMA, L. D.; QUEIROZ, L. F. N. O Processo de descentralização e regionalização do SUS no contexto do pacto pela saúde. In: MACHADO, C. V.; BAPTISTA, T. W. F.; LIMA, L. D. (Org.). Políticas de saúde no Brasil, continuidades e mudanças. Rio de Janeiro: Fiocruz, 2012. p. 229-252.

MACHADO, C. V. O Modelo de intervenção do Estado na saúde: notas sobre a atuação federal. In: MACHADO, C. V.; BAPTISTA, T. W. F.; LIMA, L. D. (Org.). Políticas de Saúde no Brasil, continuidades e mudanças. Rio de Janeiro: Fiocruz, 2012. p. 117-148. 
MALTA, D. C. et al. A implementação das prioridades da Política Nacional de Promoção da Saúde, um balanço, 2006 a 2014. Ciênc. Saúde Coletiva, Rio de Janeiro, v. 19, n. 11, p. 4301-4312, nov. 2014.

NORONHA, J. C.; LIMA, L. D.; MACHADO, C. V. Sistema Único de Saúde - SUS. In: GIOVANELLA, L. et al. (Org.). Políticas e sistemas de saúde no Brasil. Rio de Janeiro: Fiocruz, 2008. p. 435-472.

SCHMIDT, M. I. et al. Chronic non-communicable diseases in Brazil: burden and current challenges. The Lancet, Londres, v. 377, n. 9781, p. 1949-1961, 2011.

SOARES, C. L. M.; PAIM, J. S. Aspectos críticos para a implementação da política de saúde bucal no Município de Salvador, Bahia, Brasil. Cad. Saúde Pública, Rio de Janeiro, v. 27, n. 5, p. 966-974, maio 2011.

VASCONCELOS, C. M. et al. O Sistema Único de Saúde. In: CAMPOS, G. W. S. et al. Tratado de saúde coletiva. 2. ed. São Paulo/Rio de Janeiro: Hucitec/Fiocruz, 2009.
VENÂNCIO, S. I. et al. Análise de implantação da rede amamenta Brasil: desafios e perspectivas da promoção do aleitamento materno na atenção básica. Cad. Saúde Pública, Rio de Janeiro, v. 29, n. 11, p. 2261-2274, nov. 2013.

VIANA, A. L. d'Á.; LEVOCOCITZ, E. Proteção social: introduzindo o debate. In: VIANA, A. L. d'Á.; ELIAS, P. E. M.; IBANEZ, N. (Org.). Proteção social: dilemas e desafios. São Paulo: Hucitec, 2005.

VIANA, A. L. d'Á.; MACHADO, C. V. Descentralização e coordenação federativa: a experiência brasileira na saúde. Ciênc. Saúde Coletiva, v. 14, n. 3, p. 807-817, jun. 2009.

\footnotetext{
Recebido para publicação em maio de 2015

Versão final em outubro de 2015

Conflito de interesses: inexistente

Suporte financeiro: bolsa de mestrado acadêmico da Fapesp.

Processo número: 2013/06858-7
} 